\title{
Patient satisfaction and objective outcome assessment following mid-urethral sling procedures in women with stress urinary incontinence: $A$ prospective study
}

\section{Zinat Ghanbari}

University of Tehran

Seyedeh Belin Tavakoly Sany

Mashhad University of Medical Sciences

Maryam Hajhashemi

Isfahan University of Medical Sciences

Nahid Radnia

Hamedan University of Medical Science

Arezoo Orooji

Mashhad University of Medical Sciences

Taraneh Mohajer ( $\sim$ dr.taraneh57@gmail.com )

University of Tehran

\section{Research article}

Keywords: Mid-urethral sling, stress urinary incontinence, voiding dysfunction, postoperative voiding dysfunction

Posted Date: July 12th, 2019

DOI: https://doi.org/10.21203/rs.2.11250/v1

License: (c) (i) This work is licensed under a Creative Commons Attribution 4.0 International License. Read Full License 


\section{Abstract}

Background Mid-urethral sling (MUS) operations are currently a recognized as the most popular and effective procedures for the surgical treatment of stress urinary incontinence (SUI) among women population. However, data reporting a long-term outcome is still equivocal, and thus, the data available are limited. This study aims to examine the efficacy of MUS operations after implantation for the treatment of women SUI during 3-years follow up. Method A prospective study was conducted in referral centers in the Tehran University of Medical Science, Emam Khomeini Hospital, Iran. 105 patients with urodynamically proven pure SUI or mix incontinence treated by MUS surgeries (TOT-TVT) were included. Patients with history of prior anti-incontinence surgery and abdominal radical pelvic surgery were excluded. Data regarding objective cure rates, subjective outcomes (Urogenital Distress Inventory Questionnaire-Short Form, Surgical Satisfaction Questionnaire) were collected during follow-up. We analyzed the preoperative parameters by univariate and multivariate analysis was conducted to investigate outcomes. Results One hundred five women had MUS implantation. At 4-years follow-up, 101 women (95\%) were available for the assessment. We find evidence of significant improvement in urinary symptoms, objective cure rates and patient satisfaction after operation. At 3-years follow up, 80 of 101 women $(82.17 \%)$ indicated themselves cured. Similarly, at 3-years evaluation, 83 of 101 women $(82.17 \%)$ were satisfied or very satisfied, not showing visible urine leakage; and a state that did not need interval surgery. The multivariate analysis of the preoperative parameters revealed that age, VD, absences of urgency symptoms were the parameters related independently to the surgical success and patient's satisfaction and Qmax was well associated with postoperative voiding dysfunction (PVD). Conclusion The 4-years findings of this study suggest that MUS surgeries are an effective procedures for the treatment of SUl.

\section{Abstract}

Background: Mid-urethral sling (MUS) operations are currently a recognized as the most popular and effective procedures for the surgical treatment of stress urinary incontinence (SUI) among women population. However, data reporting a long-term outcome is still equivocal, and thus, the data available are limited. This study aims to examine the efficacy of MUS operations after implantation for the treatment of women SUI during 3-years follow up.

Method: A prospective study was conducted in referral centers in the Tehran University of Medical Science, Emam Khomeini Hospital, Iran. 105 patients with urodynamically proven pure SUI or mix incontinence treated by MUS surgeries (TOT-TVT) were included. Patients with history of prior antiincontinence surgery and abdominal radical pelvic surgery were excluded. Data regarding objective cure rates, subjective outcomes (Urogenital Distress Inventory Questionnaire-Short Form, Surgical Satisfaction Questionnaire) were collected during follow-up. We analyzed the preoperative parameters by univariate and multivariate

analysis was conducted to investigate outcomes. 
Results: One hundred five women had MUS implantation. At 4-years follow-up, 101 women (95\%) were available for the assessment. We find evidence of significant improvement in urinary symptoms, objective cure rates and patient satisfaction after operation. At 3-years follow up, 80 of 101 women $(82.17 \%)$ indicated themselves cured. Similarly, at 3-years evaluation, 83 of 101 women (82.17\%) were satisfied or very satisfied, not showing visible urine leakage; and a state that did not need interval surgery. The multivariate analysis of the preoperative parameters revealed that age, VD, absences of urgency symptoms were the parameters related independently to the surgical success and patient's satisfaction and Qmax was well associated with postoperative voiding dysfunction (PVD).

Conclusion: The 4-years findings of this study suggest that MUS surgeries are an effective procedures for the treatment of SUI.

Keywords: Mid-urethral sling, stress urinary incontinence, voiding dysfunction, postoperative voiding dysfunction

\section{Background}

Stress urinary incontinence (SUI) (involuntary leakage of urine on exertion, laughing, coughing, and sneezing) is the most common debilitating social problem, which leads to a reduction in their quality of life [1, 2]. It is associated with significant sexual dysfunction, loss of independence, physical morbidity, and a reduction in psychological well-being, with consequent decreased participation in domestic and social activities $[2,3]$.

Over the years, the mid-urethral sling (MUS) operation has become the most common female SUI treatment owing to its long-term high success rate, safety, easiness and low complication rates is grade A for surgical treatment of MUS as the latest technical advances, which are more effective and simpler than previous anti-incontinence operations [1, 4]. European Association of Urology (EAU) guidelines recently recommended Tension-free midurethral sling is grade A for surgical treatment of MUS as the latest technical advances, which are more effective and simpler than previous anti-incontinence operations [5, 6]. Theoretically, in a MUS operation, a tape is placed underneath the urethra, when the woman coughs, the urethra is compressed, thus preventing urine leakage. Two main methods are used to carry out this operation, either by placing a tape behind the pubic bone through groin ('transobturator'), or through the abdomen ('retropubic') [4]. Although, several studies have reported the success rate of MUS surgery for SUl, some patients experience complications following this operation such as de novo urgency or mesh erosion, recurrent SUI and postoperative voiding dysfunction (PVD) $[1,2,7]$.

The effect of MUS operation on the PVD of the patient is clinically one of the major complication in determining failure or success $[7,8]$. Although VD is a well-recognized complication, the methods for and definitions of evaluating VD are still controversial in the literature because the symptoms and incidence of VD is variable. However, most of literature represented the association of any subjective complaint of abnormal voiding such as: decreased flow rate, hesitancy, Intermittency, spraying (splitting), incomplete bladder emptying, straining to void and need to immediately re-void and objective significant postvoid 
residual (PVR), ouroflowmetry changes [1, 2]. In general, International Urogynaecological association and the International Continence Society defined VD as "incomplete micturition and /or abnormally slow based on urodynamic and symptoms investigations"[9]. Therefore, PVD has a profound effect on patient's satisfaction and quality of life. In addition, the data about the VD in women following this operation are sparse and scarce in terms of objective and subjective outcomes and patient's satisfaction, likely due to its lower incidence and various definitions of PVD. Women only able to use some urodynamic parameters to describe VD. Such conflict in definition, could lead confusion during the explanation of informed consent. Further, it can cause a clinician to overstate the risk of post-VD or overlook the patients' complaint $[2,10]$.

In this observational, retrospective study in a selected patient group, we examined long-term objective outcome and the subjective development of women with MUS implantation for SUI with follow up 4 years to evaluate the efficacy of MUS procedures. Further, we evaluated the influence of preoperative parameters potentially predicted PVD, objective postoperative cure rate, subjective development and patient-reported correction of SUI.

\section{Materials And Methods}

\section{Selection of Participants}

From May 2013 to June 2016, a total of 165 consecutive patients underwent the MUS procedure (TOTTVT) alone or with another pelvic reconstructive operation were evaluated, of whom 60 refused to participate or did not meet the inclusion criteria. These patients were prospectively reviewed after approval from the Tehran University of Medical Science, Emam Khomeini Hospital. General surgical history data were obtained from the electronic medical records. In this study, patient were included if they complain of stress incontinence and their urodynamic findings show pure SUI or mix incontinence with prominent SUI. Patients were also excluded from the analysis if they had a primary abdominal radical pelvic surgery, neurological disorders, psychiatric disorders or failed anti incontinence surgery and refused research consent. The participants were informed orally and the possibility of withdrawing at any time. Written informed consent was obtained from the participants.

\section{Preoperative Evaluation}

In this study, all participants were auscultated by a fellowship of pelvic floor dysfunction to evaluate voiding symptoms, pelvic organ prolapse quantification (POP-Q) and stress or urge incontinency as well as full- multichannel urodynamic test was performed for all participants before MUS operation. The eligible voiding dysfunction symptoms included slow stream, intermittency, hesitancy, incomplete bladder emptying, post-micturition leakage and position-dependent micturition, need to immediately re-void[7].

The urodynamic study included pressure-flow study, cystometrogram, and measurement of leak point pressure, urethral closure pressure and uroflowmetry [11]. We used an MMS International model solar silver urodynamic system to perform the urodynamic study (MMS International, Dover, NH, USA). 
Generally, correction of SUI was conducted under spinal or general anesthesia with the use of TOT or TVT techniques (American Medical Systems, Minnetonka, MN). The surgery procedures were described by Mellier et al. $[12,13]$. The tape tension was adjusted by using a metzenbaum scissors or a right angle clamp between the urethra and tape. Then, a Foley catheter was indwelled at the end of the operation, and the petrolatum gauze was used to pack vagina. On the first day after operation, the vaginal gauze and Foley catheter were removed and voiding trials and PVR measurement were conducted before discharge [13].

\section{Postoperative Evaluations}

Postoperative evaluations were mandatory at 12 months and 36 months, and every follow-up visits were performed by a fellowship of pelvic floor dysfunction. These visits included physical examination, medical history, voiding symptoms associated with surgery (voiding dysfunction and denvo urge), POP-Q, vaginal erosion of mesh, simple uroflowmetry test, PVR test and evaluation of subjective satisfaction and long-term complications. Outcome assessment was based on response to validated questionnaires or checklist. All definitions and methods followed those of the standards recommended by the International Continence Society and Strengthening the Reporting of Observational Studies in Epidemiology statement [14].

In this study, the objective cure of SUI (surgical success) was considered as the absence of urine leakage during the stress test. Thus, to achieve surgical success, patients must to report that urinary symptoms after SUI release were "a "much better" on the Urogenital Distress Inventory (UDI 6) questionnaire [15], not showing visible urine leakage at in usual activities or in postoperative stress provocation test; and a state that did not need interval surgery. Postoperative VD was considered as patients' subjective complaints (slow stream, feeling of incomplete emptying, and straining, intermittency, hesitancy, post-micturition leakage), as well as storage symptoms with PVR more than $100 \mathrm{ml}$ (or more than one third of urine volume), and postoperative de novo overactive bladder (OAB) was defined as urge incontinence and novo urgency [16]. To define the subjective outcomes, the UDI 6 global assessment questions was evaluated "how much patient were bothered by urinary condition and VD", which was scored on a 4-point Likert scale from "Not at all" to "a greatly bothered'. Failure of voiding was defined as PVR was more than 100 $\mathrm{mL}$ at voiding or more than one third of voided volume. Others condition were considered as being successful in voiding trial. In addition, urgency and cough incontinence were considered for each patients if they answered "greatly bothered" or "a moderately bothered" to question 2 an 3 pf the UDI6 questionnaire, "Urine leakage related to the feeling of urgency? and "Urine leakage related to physical activity, coughing or sneezing?", respectively.

We used the Surgical Satisfaction Questionnaire (SSQ-8) to evaluate patient satisfaction. The SSQ-8 is a valid and reliable test and responses for the all questions ranged from "very satisfied," "satisfied," "neutral," or "unsatisfied," "very-satisfied,"[17].

\section{Data Analysis}


We used IBM SPSS ver. 19.0 (IBM Co., Armonk, NY, USA) to conduct initial descriptive statistics for all variables. Bivariate analyses and one-way analysis of variance were tested to examine significant differences among groups and continuous variables. We tested Hierarchical multiple regression analysis to analyze predictors and risk factor. The variables were entered into a regression model based on theoretical and logical considerations in present study. Statistical significance was considered for a Pvalue less than $0.05(P<0.05)$.

\section{Results}

During the study period, 105 women underwent the MUS procedure (TOT-TVT) alone or with another pelvic reconstructive operation that fulfilled the inclusion criteria were evaluated. At 3-years follow-up, 101 women (96\%) were available for the assessment and 4 women (4\%) were no longer evaluable or were lost to follow-up (Figure 1). Clinical and demographic characteristics for the all eligible participants were summarized in Table 1. Most patients were premenopausal; mean body mass index was $25.67 \mathrm{~kg} / \mathrm{m} 2$.

Using bivariate analyses, our data do not show any significant differences $(p>0.05)$ of the subjective outcomes over the follow-up period even assuming all missing data as cured or as failures (Table 2). Of note, 3 years after surgery, 83 of 101 women $(82.17 \%)$ reported themselves cured. Similar to the subjective data, at the 3-years follow-up, 80 of 101 patients $(79.2 \%)$ were also objectively cured. No significant change of objective cure rates was found during follow up $(p=0.6)$ (Table 2). All preoperative and follow-up subjective symptoms were compared among 105 patients (Table 3). Between two groups, we found evidence of significant improvement $(p<0.05)$ in the urinary symptoms, cough test, and urgency condition after operation. No significant mesh erosion ( $97.14 \%$ success outcome) was also registered in our study population over follow up. Further, 79.04\% patients were satisfied or very satisfied, not showing visible urine leakage at in usual activities or in postoperative stress provocation test; and a state that did not need interval surgery.

Table 4 reported multivariate analysis of preoperative parameters potentially predicted change of PVD, cure of USI and patient's satisfaction after operation. This analysis demonstrated that age, absence of documented urgency symptom and VD were the parameters related independently to the surgical success and patient's satisfaction but other factors were not significant. Notably, our finding showed that age, Qmax and VD was also appropriate predictors for PVD.

\section{Discussion}

This work reports the combination of objective and subjective outcomes of the MUS procedure (TOT-TVT) at 3-years follow-up. We found that MUS is effective and safe procedure. The 3-years subjective and objective outcomes were $82.17 \%$ and $79.2 \%$, respectively. This showed that MUS procedure has longlasting effectiveness. A recent meta-analysis and systematic review showed that transobturator MUS (TOTs) and retropubic MUS (TVT) have similar subjective and objective cure rates in the medium and 
long term because MUS is effectively used the gold-standard methods for the surgery treatment of women SUI $[7,8,18]$.

Very few studies have evaluated medium- to long term outcomes of MUS procedure, particularly TVT-O routs[8]. In most of these studies, women with urodynamically proven detrusor overactivity (DO) and overactive bladder $(\mathrm{OAB})$ symptoms were included that lead to decrease the reliability of the results [19, 20]. Athanasiou et al reported 7 -years subjective and objective cure rates of $83 \%$ and $81 \%$, respectively [21]. The slightly lower subjective and objective cure rates in our study could be due to the fact that we assessed 105 consecutive women who underwent a MUS procedure without or with concomitant pelvic reconstructive operation. Several studies reported that women with mixed urinary incontinence showed a higher risk of objective and subjective failure. In a prospective, Costantini et al indicated objective and subjective cure rates of $70 \%$ and $59 \%$ among 47 women included in the TOT group [22]. A limitation of this study could be that patient with mixed urinary incontinence were included, which likely affected the cure rates $[8,22]$. It is noteworthy that results of this study demonstrated 3 -years cure rate that is comparable with various studies that reported the MUS procedure with much longer follow-up $[7,8]$.

The progression of de novo OAB symptoms is one of the most largely significant postoperative complications of MUSs. Recent studies have reported de novo urgency rates ranging from $4-33 \%$ after MUSs operation $[16,23]$. However, very few medium- or long run studies are available on the relation between $O A B$ symptom onset and MUSs procedures. Previous study have reported onset of $O A B$ symptom (urge incontinence and novo urgency) in $24 \%$ of patients at 1-years follow-up and $19 \%$ of patients at 5-yr follow-up [24]. In this study, at 3 years follow-up after MUS surgeries, we observed a considerable reduction in the novo urgency rates $(25 \%)$. These results would be useful for preoperative counseling because the postoperative $O A B$ symptoms have significant effect on patients' quality of life and their satisfaction after MUS surgery.

We have also considered different possible demographic and clinical parameters to assess independent predictor for outcomes after operation. Here, women who were younger, low rate of VD symptoms and lacked OAB symptoms before operation were more likely associated with surgical success and patients' satisfaction. Notably, our findings suggest that Qmax value is significant predictor of the PVD. No studies on preoperative urodynamic find this association between Qmax values and an increased risk of VD. As previously reported, in many such cases, Qmax values were similar among patient with VD and those without VD [25]. However, recent studies demonstrated a significant association between PVD and Qmax $<15 \mathrm{ml} / \mathrm{s}$ [10]. Another recent studies reported that of 625 patients who underwent the various type of MUS surgeries (TOT, TVT-secure, TVT, TVT-obturator), 163 (26\%) patients showed PVD, and Qmax was the only risk factor for $\mathrm{PVD}[26,27]$. From a clinical standpoint, our findings is important for surgeons and preoperative patient counseling to mange patient complications and to select the best approach for sling release. As matter of fact, we investigated the risk factors that could be used in predicting PVD. The results showed that although some patients complain of PVD, they had normal clinical assessments (QMAX, PVR and flowmetry). Moreover patients satisfaction after surgery were not decreased. Points of strength of this study are the following: (1) assessment of a large number of patients; (2) use of validated 
questionnaires to assess the objective and subjective outcomes; (4) the rate of loss to follow-up was very low; (5) significant improvement in outcomes.

Limitations of this study must be explained, we did not use validated quality-of-life questionnaires because no validated quality-of-life questionnaire exists in Iran. However, we used the validated and standardized Urogenital Distress Inventory (UDI 6) form and Surgical Satisfaction Questionnaire (SSQ-8) to examine results of the MUS surgeries. Likewise, given that the outcomes of interest were patientreported improvement and satisfaction, this work could be inherently vulnerable to respondent bias. Therefore, further studies, potentially in multicenter cohorts, are required to help define the optimal management strategy.

\section{Conclusions}

The 3-year results of this study showed that MUS is a highly safe option and effective for the treatment of women SUI. The subjective and objective cure rates were both high. The identification of potential predictors suggest that preoperative parameters, including younger age, lack of OAB symptoms and VD could be used to predict cure of USI and patients' satisfaction, which can provide clinician with additional information for preoperative counseling. In addition, Qmax is considered was significant predictor of PVD, which may help surgeons to identify proper surgical candidates.

\section{Abbreviations}

UDI 6: Urogenital Distress Inventory; SSQ-8: Surgical Satisfaction Questionnaire; DO: detrusor overactivity; OAB: overactive bladder; SSQ-8: Surgical Satisfaction Questionnaire; POP-Q: pelvic organ prolapse quantification PVR: postvoid residual; PVD: postoperative voiding dysfunction; EAU: European Association of Urology; SUl: stress urinary incontinence; MUS Mid-urethral sling

\section{Declarations}

\section{Acknowledgements}

The author wishes to express her gratitude towards the vice president of research in Tehran University of Medical Sciences, Iran

\section{Authors' Contributions:}

$\mathrm{TM}, \mathrm{NR}, \mathrm{MH}$ contributed to the design of the analysis plan, the acquisition of data and data interpretation. SBTS and AO contributed to draft the article and statistical analysis. ZGH contributed to the design of the analysis plan and supported the data interpretation.

\section{Funding}

This study was supported by grants from the Tehran University of Medical Science, Iran. 


\section{Availability of data and materials}

The datasets generated and analyzed during the current study are available from the corresponding author on reasonable request.

\section{Ethics Approval and Consent to Participate}

This study was conducted after the approval and permission of Tehran University of Medical Sciences Research Committee 33461-246516 and was conducted with consideration of Helsinki Declaration in all phases of the study. Confidential data treatment was guaranteed. Written informed consent was obtained from the participants. Availability of data and materials Data from this study will not be openly available until planned publication outputs have been completed.

\section{Consent for publication}

All patients including the index case signed a consent form for publication.

\section{Competing Interests}

The author(s) declare that they have no competing interests.

\section{References}

1. Chae, J.Y., et al., Effect of Preoperative Low Maximal Flow Rate on Postoperative Voiding Trials after the Midurethral Sling Procedure in Women with Stress Urinary Incontinence. LUTS: Lower Urinary Tract Symptoms, 2017.

2. Aponte, M.M. and V.W. Nitti, Sling Procedures: Voiding Dysfunction after Stress Urinary Incontinence Surgery. Gynecologic and Obstetric Surgery: Challenges and Management Options, 2016: p. 318-320.

3. Salonia, A., et al., Sexual dysfunction is common in women with lower urinary tract symptoms and urinary incontinence: results of a cross-sectional study. European urology, 2004. 45(5): p. 642-648.

4. Ulrich, D., et al., Quality of life and objective outcome assessment in women with tape division after surgery for stress urinary incontinence. PloS one, 2017. 12(3): p. e0174628.

5. Lucas, M.G., et al., EAU guidelines on surgical treatment of urinary incontinence. European urology, 2012. 62(6): p. 1118-1129.

6. Chapple, C.R., et al., Consensus statement of the European Urology Association and the European Urogynaecological Association on the use of implanted materials for treating pelvic organ prolapse and stress urinary incontinence. European urology, 2017. 72(3): p. 424-431.

7. Braga, A., et al., Tension-free vaginal tape for treatment of pure urodynamic stress urinary incontinence: efficacy and adverse effects at 17-year follow-up. BJU international, 2018. 
8. Serati, M., et al., Tension-free vaginal tape-obturator for treatment of pure urodynamic stress urinary incontinence: efficacy and adverse effects at 10-year follow-up. European urology, 2017. 71(4): p. 674679.

9. Bo, K., et al., An International Urogynecological Association (IUGA)/International Continence Society (ICS) joint report on the terminology for the conservative and nonpharmacological management of female pelvic floor dysfunction. International urogynecology journal, 2017. 28(2): p. 191-213.

10. Cho, S.-T., et al., Predictors of postoperative voiding dysfunction following transobsturator sling procedures in patients with stress urinary incontinence. International neurourology journal, 2010. 14(1): p. 26.

11. Schäfer, W., et al., Good urodynamic practices: Uroflowmetry, filling cystometry, and pressure-flow studies. Neurourology and Urodynamics: Official Journal of the International Continence Society, 2002. 21(3): p. 261-274.

12. de Leval, J., Novel surgical technique for the treatment of female stress urinary incontinence: transobturator vaginal tape inside-out. European urology, 2003. 44(6): p. 724-730.

13. Mellier, G., et al., Suburethral tape via the obturator route: is the TOT a simplification of the TVT? International Urogynecology Journal, 2004. 15(4): p. 227-232.

14. Toozs-Hobson, P., et al., An International Urogynecological Association (IUGA)/International Continence Society (ICS) joint report on the terminology for reporting outcomes of surgical procedures for pelvic organ prolapse. International urogynecology journal, 2012. 23(5): p. 527-535.

15. Uebersax, J.S., et al., Short forms to assess life quality and symptom distress for urinary incontinence in women: the Incontinence Impact Questionnaire and the Urogenital Distress Inventory. Neurourology and urodynamics, 1995. 14(2): p. 131-139.

16. Serati, M., et al., Tension-free vaginal tape for the treatment of urodynamic stress incontinence: efficacy and adverse effects at 10-year follow-up. European urology, 2012. 61(5): p. 939-946.

17. Haff, R.E., et al., The surgical satisfaction questionnaire (SSQ-8): a validated tool for assessment of patient satisfaction following surgery to correct prolapse and/or incontinence. Journal of Minimally Invasive Gynecology, 2011. 18(6): p. S49-S50.

18. Tommaselli, G.A., et al., Medium-term and long-term outcomes following placement of midurethral slings for stress urinary incontinence: a systematic review and metaanalysis. International urogynecology journal, 2015. 26(9): p. 1253-1268.

19. Cheng, D. and C. Liu, Tension-free vaginal tape-obturator in the treatment of stress urinary incontinence: a prospective study with five-year follow-up. European Journal of Obstetrics \& Gynecology and Reproductive Biology, 2012. 161(2): p. 228-231. 
20. Laurikainen, E., et al., Five-year results of a randomized trial comparing retropubic and transobturator midurethral slings for stress incontinence. European urology, 2014. 65(6): p. 1109-1114.

21. Athanasiou, S., et al., Seven years of objective and subjective outcomes of transobturator (TVT-O) vaginal tape: why do tapes fail? International urogynecology journal, 2014. 25(2): p. 219-225.

22. Costantini, E., et al., Long-term efficacy of the trans-obturator and retropubic mid-urethral slings for stress urinary incontinence: update from a randomized clinical trial. World journal of urology, 2016. 34(4): p. 585-593.

23. Novara, G., et al., Complication rates of tension-free midurethral slings in the treatment of female stress urinary incontinence: a systematic review and meta-analysis of randomized controlled trials comparing tension-free midurethral tapes to other surgical procedures and different devices. european urology, 2008. 53(2): p. 288-309.

24. Serati, M., et al., TVT-O for the treatment of pure urodynamic stress incontinence: efficacy, adverse effects, and prognostic factors at 5-year follow-up. European urology, 2013. 63(5): p. 872-878.

25. Lemack, G.E., et al., Normal preoperative urodynamic testing does not predict voiding dysfunction after Burch colposuspension versus pubovaginal sling. The Journal of urology, 2008. 180(5): p. 20762080.

26. Salin, A., et al., Identification of risk factors for voiding dysfunction following TVT placement. European urology, 2007. 51(3): p. 782-787.

27. Jang, H.A., J.H. Bae, and J.G. Lee, Incidence and risk factors of postoperative de novo voiding dysfunction following midurethral sling procedures. Korean Journal of Urology, 2009. 50(8): p. 762-766.

\section{Figures}




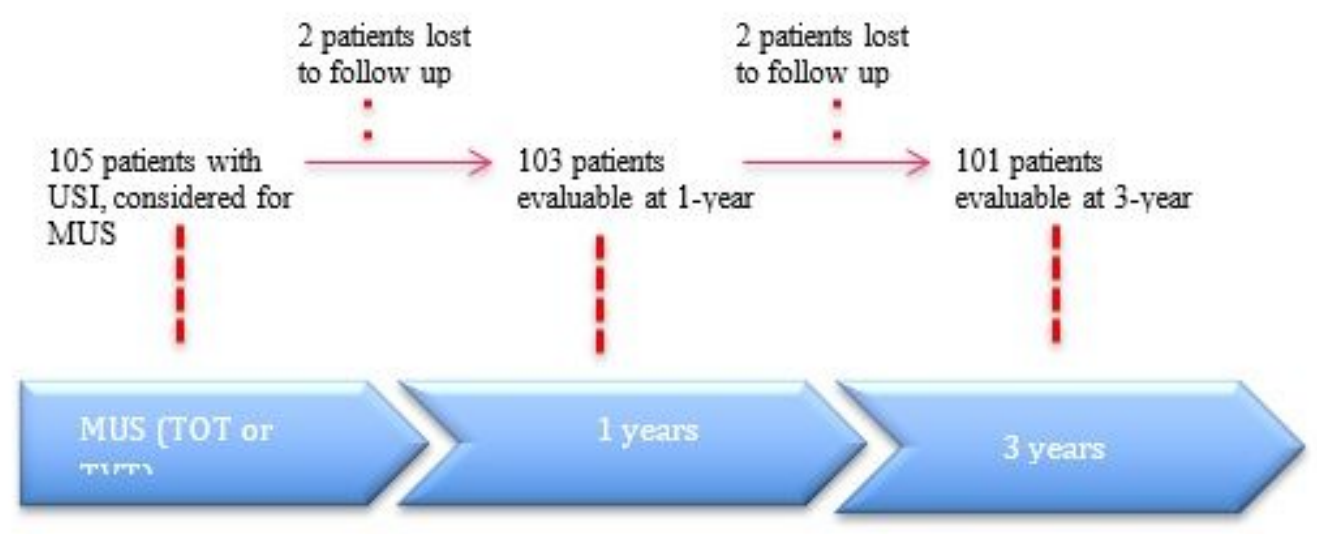

Figure. 1 - Progress of patients across the study period

\section{Figure 1}

\section{Supplementary Files}

This is a list of supplementary files associated with this preprint. Click to download.

- Tables.pdf 